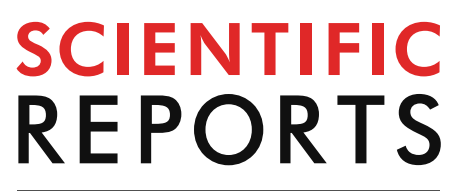

natureresearch

Check for updates

\title{
Incidence and impact
} of extra-pulmonary organ failures on hospital mortality in acute exacerbation of idiopathic
pulmonary fibrosis Yi Zhuang ${ }^{1,2}$, Ying Zhou ${ }^{1,2}$, Xiaohua Qiu ${ }^{1}$, Yonglong Xiao ${ }^{1}$, Hourong Cai ${ }^{1}$ \& Jinghong Dai ${ }^{1 凶}$

To evaluate hospital mortality and associated risk factors for acute exacerbations of idiopathic pulmonary fibrosis (AEIPF). Emphases were put on incidence and impact of extra-pulmonary organ failures. Patients diagnosed with AEIPF from July 2014 to September 2018 were enrolled. Clinical data were collected. Acute physiology and chronic health evaluation II (APACHE II) and simplified acute physiological score II (SAPS II) were calculated. Extra-pulmonary organ failures were diagnosed upon criteria of sequential organ failure assessment (SOFA). Forty-five patients with AEIPF were included. Eighteen patients (40.0\%) developed extra-pulmonary organ failures, and 25 patients $(55.6 \%)$ died during hospitalization. Serum C-reactive protein (CRP) $(p=0.001)$, SAPS II $(p=0.004)$, SOFA $(p=0.001)$ were higher, whereas arterial oxygen pressure $\left(\mathrm{PaO}_{2}\right) /$ fractional inspired oxygen $\left(\mathrm{FiO}_{2}\right)(p=0.001)$ was lower in non-survivors than survivors. More non-survivors developed extra-pulmonary organ failures than survivors $(p=0.002)$. After adjustment, elevated serum CRP (OR 1.038, $p=0.049)$ and extrapulmonary organ failure (OR 13.126, $p=0.016$ ) were independent predictors of hospital mortality in AEIPF. AEIPF had high hospital mortality and occurrence of extra-pulmonary organ failure was common. Elevated serum CRP and extra-pulmonary organ failure had predictive values for mortality.

Idiopathic pulmonary fibrosis (IPF) is a chronic, progressive and fatal fibrosing interstitial pneumonia with unknown etiology ${ }^{1}$. The clinical course of IPF is highly heterogeneous and unpredictable. For some patients, the disease remains stable or progresses slowly over years. But a small amount of patients may develop sudden exacerbations of respiratory function impairment, referred to as acute exacerbations of idiopathic pulmonary fibrosis $(\mathrm{AEIPF})^{2}$, resulting in refractory hypoxemia and respiratory failure. AEIPF is the leading cause of death in IPF, with the hospital mortality up to $60 \%{ }^{2,3}$.

AEIPF shared similar pathophysiological characteristics and clinical need with another severe condition, acute respiratory distress syndrome (ARDS) ${ }^{4}$. A majority of patients with AEIPF needed admission to intensive care unit (ICU) for severe complications and multiple organ failures. AEIPF was also considered as an inflammation associated disease ${ }^{5}$. Despite the high hospital mortality rates, the prognostic factors for short-term mortality in patients with AEIPF remained uncertain. Few studies described the conditions of extra-pulmonary organs in patients with AEIPF. Therefore, we conducted this retrospective study in an interstitial lung disease center in China, to investigate the hospital mortality and associated risk factors of hospital mortality in patients with AEIPF. Acute physiology and chronic health evaluation II (APACHE II $)^{6}$, simplified acute physiological score II (SAPS II) ${ }^{7}$ and sequential organ failure assessment (SOFA) system ${ }^{8}$ were used to evaluate the organ conditions and assess the incidence and impact of extra-pulmonary organ failures on hospital mortality in AEIPF.

\footnotetext{
${ }^{1}$ Department of Pulmonary and Critical Care Medicine, Nanjing Drum Tower Hospital, Clinical College of Nanjing Medical School, No. 321 Zhongshan Road, Nanjing 210008, Jiangsu, China. ${ }^{2}$ These authors contributed equally: Yi Zhuang and Ying Zhou. ${ }^{\square}$ email: daijinghong@nju.edu.cn
} 


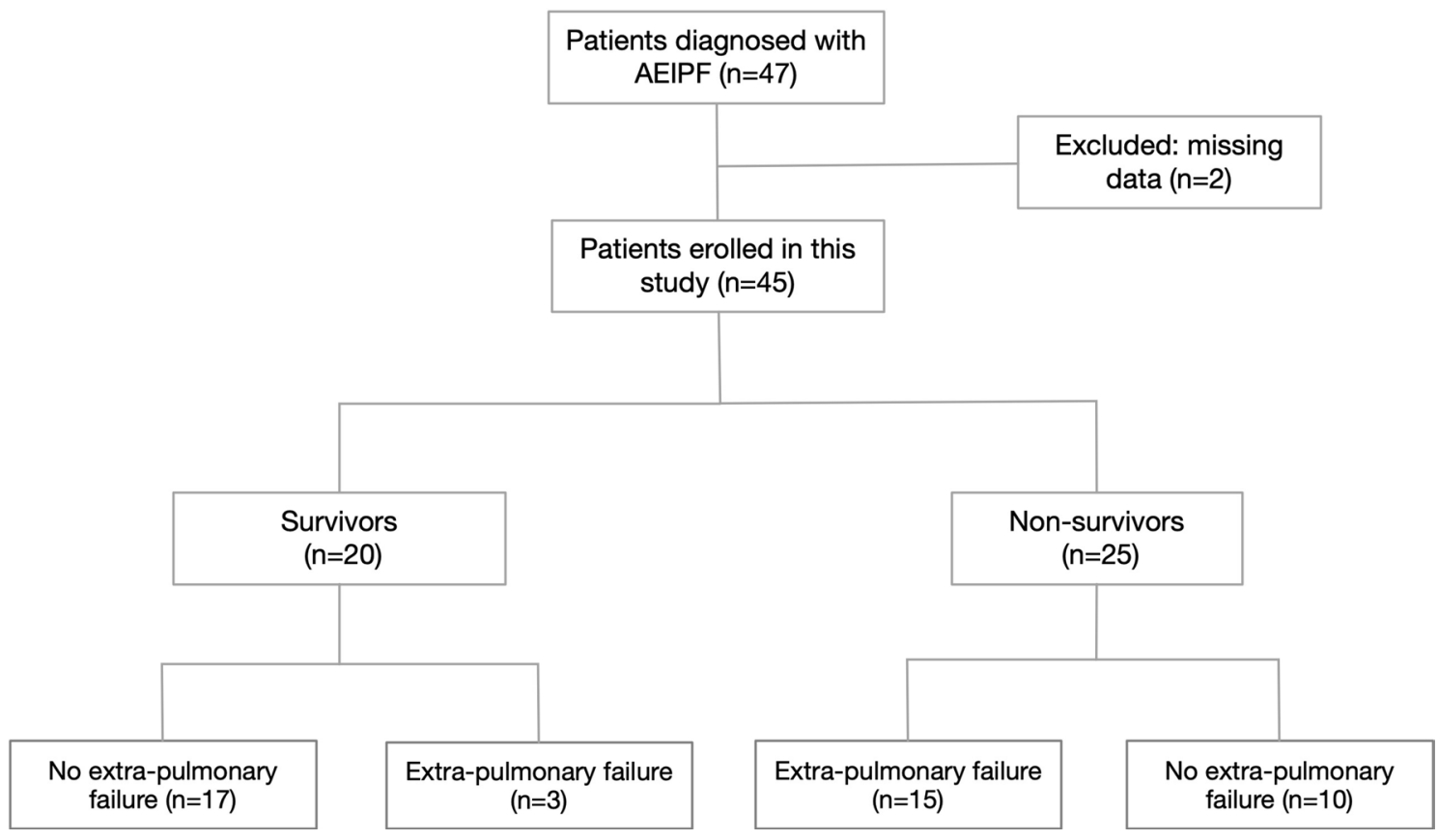

Figure 1. Flowchart of patients' selection. AEIPF = acute exacerbation of idiopathic pulmonary fibrosis.

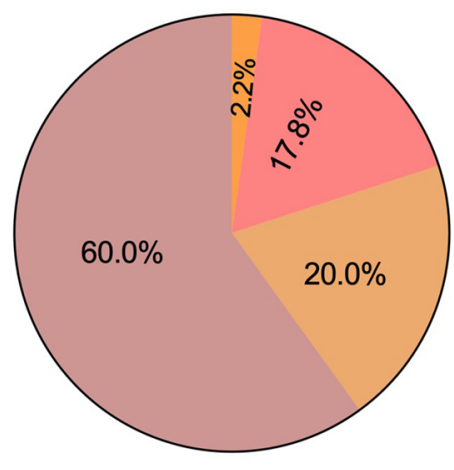

\section{Three extra-pulmonary organ failures \\ Two extra-pulmonary organ failures \\ One extra-pulmonary organ failure \\ No extra-pulmonary organ failure}

Total $=45$

Figure 2. A breakdown of presence of extra-pulmonary organ failure in AEIPF patients. Of the 45 patients, one patient $(1 / 45,2.2 \%)$ combined with three extra-pulmonary organ failures, eight patients $(8 / 45,17.8 \%)$ had two extra-pulmonary organ failures, nine patients $(9 / 45,20.0 \%)$ had one extra-pulmonary organ failure, and 27 patients $(27 / 45,60.0 \%)$ combined with no extra-pulmonary organ failure. AEIPF $=$ acute exacerbation of idiopathic pulmonary fibrosis.

\section{Results}

Patients inclusion. There were 47 consecutive patients diagnosed with AEIPF in our center during the study period. Two patients missing follow-up data were excluded. Hence, a total of 45 patients were enrolled (Fig. 1). They were 36 males and 9 females with a mean age of $66.6 \pm 9.0$ years old (range $42-82$ years old). Twenty-two (48.9\%) patients were smokers. They developed AE in 21.9 \pm 17.6 months (range 0-84 months) from the diagnosis of IPF. Before the occurrence of AEIPF, 14 patients (31.1\%) had received corticosteroids treatment for $11.9 \pm 11.3$ months (range 3-36 months) and 2 patients (4.4\%) were on pirfenidone or nintedanib therapy for 5.5 \pm 2.5 months (range, 3-8 months).

At admission, the mean arterial oxygen pressure $\left(\mathrm{PaO}_{2}\right) /$ fractional inspired oxygen $\left(\mathrm{FiO}_{2}\right)$ was $158.0 \pm 60.3 \mathrm{mmHg}$ (range 45.0-282.8 $\mathrm{mmHg}$ ). Extra-pulmonary organ failures were observed in 18 patients $(40.0 \%)$. Among them, 13 patients $(13 / 18,72.2 \%)$ had acute cardiovascular failure, and 5 of them were treated with vasopressors infusion. Six patients $(6 / 18,33.3 \%)$ had acute liver failure and 4 patients $(4 / 18,22.2 \%)$ had acute kidney failure. One patient $(1 / 18,5.6 \%)$ had 3 extra-pulmonary organ failures, 8 patients $(8 / 18,44.4 \%)$ had 2 extra-pulmonary organ failures, and 9 patients $(9 / 18,50.0 \%)$ had 1 extra-pulmonary organ failure (Fig. 2). High-dose corticosteroids (0.5-1 g/d methylprednisolone or its equivalent) for 3-5 days was used in 26 patients $(57.8 \%)$ after diagnosis. Non-invasive ventilation (NIV) was used in 25 patients (55.6\%), and 2 of them were 


\begin{tabular}{|c|c|c|c|}
\hline Variables & Non-survivors $(n=25)$ & Survivors $(n=20)$ & $p$ \\
\hline Age, years & $68.2 \pm 9.0$ & $64.7 \pm 8.8$ & 0.197 \\
\hline Male, n (\%) & $20(80.0)$ & $16(80.0)$ & $>0.999$ \\
\hline Smoker, n (\%) & $13(52.0)$ & $9(45.0)$ & 0.641 \\
\hline Pre-admission steroid usage, n (\%) & $6(24.0)$ & $8(40.0)$ & 0.249 \\
\hline Pirfenidone/ nintedanib usage, n (\%) & $2(8.0)$ & $0(0.0)$ & 0.495 \\
\hline Duration from diagnosis of IPF to AE, months & $20.0 \pm 19.5$ & $24.2 \pm 15.1$ & 0.432 \\
\hline Fever, n (\%) & $9(36.0)$ & $7(35.0)$ & 0.944 \\
\hline $\mathrm{PaO}_{2} / \mathrm{FiO}_{2}, \mathrm{mmHg}$ & $131.7 \pm 51.3$ & $190.9 \pm 55.3$ & 0.001 \\
\hline $\mathrm{WBC},{ }^{\star} 10^{9} / \mathrm{L}$ & $12.0 \pm 5.7$ & $9.7 \pm 2.7$ & 0.101 \\
\hline CRP, mg/L & $66.8 \pm 53.6$ & $19.6 \pm 18.4$ & 0.001 \\
\hline $\mathrm{LDH}, \mathrm{U} / \mathrm{L}$ & $506.8 \pm 204.7$ & $469.7 \pm 146.1$ & 0.108 \\
\hline \multicolumn{4}{|l|}{ HRCT pattern, $n$} \\
\hline Peripheral/multifocal/diffuse & $4 / 6 / 15$ & $3 / 4 / 13$ & 0.936 \\
\hline Presence of extra-pulmonary organ failure, $\mathrm{n}(\%)$ & $15(60.0)$ & $3(15.0)$ & 0.002 \\
\hline APACHE II score & $17.6 \pm 3.9$ & $15.3 \pm 4.5$ & 0.075 \\
\hline SAPS II score & $31.8 \pm 6.2$ & $26.8 \pm 4.9$ & 0.004 \\
\hline SOFA score & $3.9 \pm 1.2$ & $2.8 \pm 0.7$ & 0.001 \\
\hline \multicolumn{4}{|l|}{ Treatment, n (\%) } \\
\hline High-dose steroids & $17(68.0)$ & $9(45.0)$ & 0.121 \\
\hline NIV & $22(88.0)$ & $3(15.0)$ & $<0.001$ \\
\hline IMV & $2(8.0)$ & $0(0.0)$ & 0.495 \\
\hline Lengths of stay in RICU, days & $9.2 \pm 8.4$ & $8.7 \pm 10.5$ & 0.135 \\
\hline Lengths of stay in hospital, days & $11.7 \pm 8.9$ & $19.5 \pm 7.0$ & 0.001 \\
\hline
\end{tabular}

Table 1. Comparison of clinical characteristics between non-survivors and survivors in patients with AEIPF. AE-IPF = acute exacerbation of idiopathic pulmonary fibrosis; $\mathrm{PaO}_{2}=$ arterial oxygen pressure; $\mathrm{FiO}_{2}=$ fractional inspired oxygen; $\mathrm{WBC}=$ white blood cell; $\mathrm{CRP}=\mathrm{C}$-reactive protein; HRCT = high-resolution computed tomography; APACHE II = Acute physiology and chronic health evaluation II; SAPS II = simplified acute physiological score II; SOFA II = sequential organ failure assessment; NIV = non-invasive mechanical ventilation; IMV = Invasive mechanical ventilation; RICU = respiratory intensive care unit.

performed invasive mechanical ventilation (IMV). Twenty-five patients died in the hospital with a mortality rate of $55.6 \%(25 / 45)$ and 20 patients discharged. The mean length of hospital stay was $14.7 \pm 8.8$ days (range $3-35$ days). Thirty-four patients $(75.6 \%)$ had stayed in respiratory intensive care unit (RICU) for $10.1 \pm 9.9$ days (range $2-35$ days).

\section{Comparison of clinical characteristics between survivors and non-survivors}

As presented in Table 1, serum C-reactive protein (CRP) level $(66.8 \pm 53.6 \mathrm{mg} / \mathrm{L}$ vs. $19.6 \pm 18.4 \mathrm{mg} / \mathrm{L}, p=0.001)$, SAPS II score $(31.8 \pm 6.2$ vs. $26.8 \pm 4.9, p=0.004)$ and SOFA score $(3.9 \pm 1.2$ vs. $2.8 \pm 0.7, p=0.001)$ were higher in non-survivors than those in survivors, whereas $\mathrm{PaO}_{2} / \mathrm{FiO}_{2}(131.7 \pm 51.3$ vs. $190.9 \pm 55.3, p=0.001)$ was lower in non-survivors than that in survivors. Non-survivors were more likely to develop extra-pulmonary organ failures than survivors $(60.0 \%$ vs. $15.0 \%, p=0.002)$. More non-survivors received NIV than survivors $(88.0 \%$ vs. $15.0 \%$, $p<0.001)$. No significant differences were observed in age, gender, smoking status, chest high-resolution computerized tomography (HRCT) pattern, APACHE II score or high-dose steroids usage between the two groups.

\section{Risk factors on hospital mortality}

In univariate analysis, $\mathrm{PaO}_{2} / \mathrm{FiO}_{2}(\mathrm{OR} 0.980 ; 95 \%$ CI $0.967-0.993 ; p=0.014)$, serum CRP (OR 1.040; 95\% CI $1.011-1.070 ; p=0.006)$, presence of extra-pulmonary organ failure (OR 8.500; 95\% CI 1.964-36.790; $p=0.004$ ), SAPS II score (OR 1.184; 95\% CI 1.039-1.349; $p=0.011$ ) and SOFA score (OR 3.585; 95\% CI 1.522-8.443; $p=0.003$ ) were associated with hospital mortality. After adjustment for age and gender, multivariate analysis revealed that an elevated serum CRP (OR 1.038; 95\% CI 1.001-1.076; $p=0.049$ ) and extra-pulmonary organ failure (OR 13.126; 95\% CI 1.608-107.161; $p=0.016)$ were independent risk factors for hospital mortality in patients with AEIPF (Table 2, Fig. 3 and Fig. 4).

\section{Discussion}

The study showed a majority (34/45, 75.6\%) of AEIPF patients had stayed in RICU with a high hospital mortality rate $(25 / 45,55.6 \%)$. Occurrence of extra-pulmonary organ failure $(18 / 45,40.0 \%)$ was common in patients with AEIPF. More non-survivors (60.0\%) developed multiple organ failures compared to survivors (15.0\%). Elevated serum CRP level and presence of extra-pulmonary organ failures were associated risk factors for hospital 


\begin{tabular}{|c|c|c|c|c|c|c|}
\hline & \multicolumn{3}{|c|}{ Univariate } & \multicolumn{3}{|c|}{ Multivariate } \\
\hline & OR & 95\% CI & $p$ & OR & 95\% CI & $p$ \\
\hline Age & 1.047 & $0.976-1.122$ & 0.199 & 0.948 & $0.838-1.072$ & 0.394 \\
\hline Sex & 0.571 & $0.131-2.491$ & 0.456 & 4.237 & $0.381-47.175$ & 0.240 \\
\hline Even smoker & 1.186 & $0.568-2.475$ & 0.650 & & & \\
\hline Pre-admission steroids usage & 0.898 & $0.342-2.358$ & 0.826 & & & \\
\hline Duration from diagnosis of IPF to AE & 0.986 & $0.953-1.021$ & 0.426 & & & \\
\hline $\mathrm{PaO}_{2} / \mathrm{FiO}_{2}$ & 0.980 & $0.967-0.993$ & 0.014 & 0.983 & $0.962-1.003$ & 0.101 \\
\hline WBC & 1.157 & $0.966-1.387$ & 0.114 & & & \\
\hline CRP & 1.040 & $1.011-1.070$ & 0.006 & 1.038 & $1.001-1.076$ & 0.049 \\
\hline LDH & 1.003 & $0.999-1.008$ & 0.124 & & & \\
\hline Presence of extra-pulmonary organ failure & 8.500 & $1.964-36.790$ & 0.004 & 13.126 & $1.608-107.161$ & 0.016 \\
\hline APACHE II score & 1.142 & 0.973-1.339 & 0.104 & & & \\
\hline SAPS II score & 1.184 & $1.039-1.349$ & 0.011 & 1.081 & $0.884-1.320$ & 0.449 \\
\hline SOFA score & 3.585 & $1.522-8.443$ & 0.003 & & & \\
\hline \begin{tabular}{|l} 
High-dose steroids \\
\end{tabular} & 2.597 & $0.769-8.775$ & 0.124 & & & \\
\hline
\end{tabular}

Table 2. Logistic regression analysis of risk factors of hospital mortality in AEIPF patients. Age, sex, $\mathrm{PaO}_{2} /$ $\mathrm{FiO}_{2}$, CRP, presence of extra-pulmonary organ failure and SAPS II score were included in the multivariate logistic regression analysis. $\mathrm{AEIPF}=$ acute exacerbation of idiopathic pulmonary fibrosis; $\mathrm{PaO}_{2}=$ arterial oxygen tension; $\mathrm{FiO}_{2}=$ fractional inspired oxygen; $\mathrm{WBC}=$ white blood cell; $\mathrm{CRP}=\mathrm{C}$-reactive protein; APACHE II = Acute physiology and chronic health evaluation II; SAPS II = simplified acute physiological score II; SOFA II = sequential organ failure assessment.

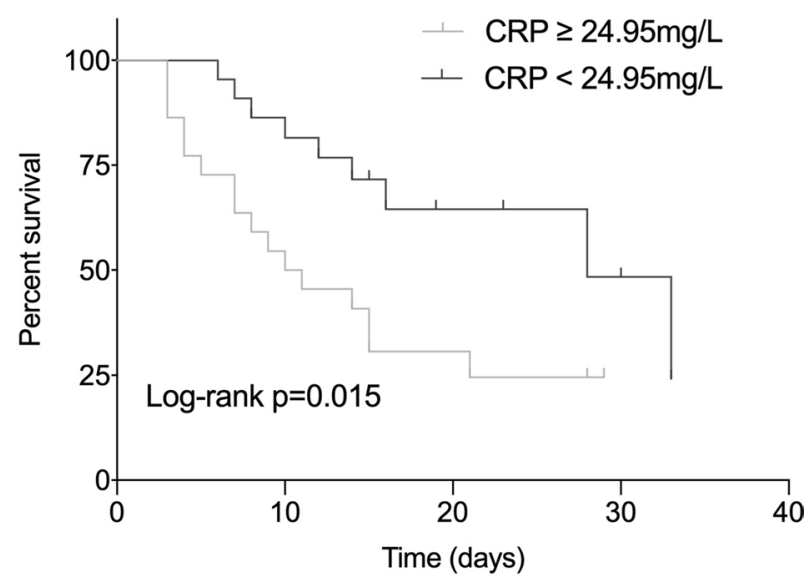

Figure 3. Kaplan-Meier survival estimate of AEIPF patients stratified according to serum CRP levels. $\mathrm{AEIPF}=$ acute exacerbation of idiopathic pulmonary fibrosis; $\mathrm{CRP}=\mathrm{C}$-reactive protein.

mortality in AEIPF. These findings implicated an association between systemic inflammation and poor outcome in AEIPF.

The outcome of AEIPF was poor, and no effective treatment options were available ${ }^{3,9-11}$. A 10-year observational study showed that hospital mortality of AEIPF was $56.9 \%$ and 3 -month mortality was $63.8 \%{ }^{11}$. Saydain et al. reported that $61 \%$ of patients with AEIPF died during hospitalization and $92 \%$ of survivors died within 2 months after discharge ${ }^{9}$. The study from Song et al. also showed that hospital mortality in AEIPF was approximately $50.0 \%^{3}$, and in patients who were admitted to ICU, it exceeded $90 \%{ }^{12}$. Various potential therapies had been reported in patients with $\mathrm{AEIPF}^{13,14}$. Unfortunately, these studies were mostly with small sample sizes and uncontrolled. Still, controversies remained over the use of corticosteroids and immunosuppressive agents in patients with AEIPF. A study from Japan demonstrated that early use of high-dose steroids could significantly increase survival rate ${ }^{15}$. But study from Papiris et al. showed corticosteroids therapy had no obvious advantage to 1 -year survival ${ }^{16}$. International guidelines made a weak recommendation for the use of corticosteroids in AEIPF $^{1}$. Results from clinical trials of nintedanib and pirfenidone suggested that IPF therapies may be helpful to prevent the development of AEIPF, but these data were from observational study ${ }^{17-19}$. A well-designed randomized clinical trial is still needed.

In the current study, CRP was identified as an independent risk factor of hospital mortality in AEIPF, which was consistent with previous studies ${ }^{3}$. Viral infection was reported to cause exacerbation of established 


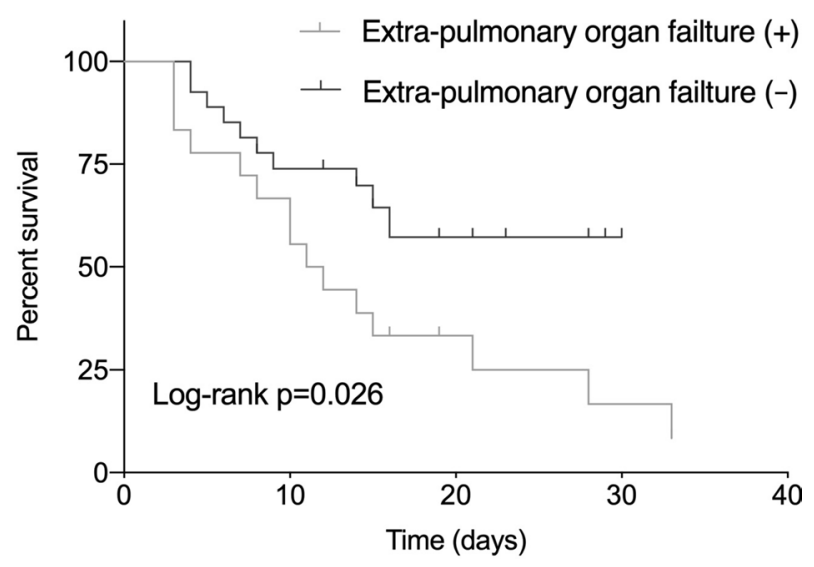

Figure 4. Kaplan-Meier survival estimate of AEIPF patients stratified according to extra-pulmonary organ failure. $\mathrm{AEIPF}=$ acute exacerbation of idiopathic pulmonary fibrosis.

pulmonary fibrosis in a murine mode ${ }^{20}$. Lung microbiome, especially Staphylococcus and Streptococcus genera, showed an association with progression of $\mathrm{IPF}^{21}$. These studies suggested that infection or inflammation could be one pathogenic mechanism contributing to the occurrence of AE. More recently, a study from Li $\mathrm{HP}$ et al. also demonstrated that inflammatory cytokines, such as IL-6, IFN- $\gamma$, MIG, IL-17 and IL-9, were increased in the serum of AE-IPF compared with stable cases ${ }^{22}$, indicating inflammation rather than an accelerated intrinsic fibrotic process played a role in pathogenesis of AEIPF.

AEIPF shared several pathophysiological characteristics with ARDS ${ }^{4}$. Previous studies had noted that extrapulmonary organ failures had increased the mortality risk of $\mathrm{ARDS}^{23}$. Sepsis syndrome/ multiple organ failures (MOFs) were the main cause of ARDS deaths ${ }^{24}$. Besides, higher APACHE II scores were reported to be associated with increased mortality in ARDS ${ }^{25}$. Similarly, this study showed that the presence of extra-pulmonary organ failure was a prognostic factor of AEIPF. It seemed that, during AEIPF, inflammation in lung triggered enhanced systemic inflammatory response leading to MOFs, and then worsened the clinical outcomes. The values of life supportive care and anti-inflammation therapy were also to be elucidated.

Notably, in our cohort, a majority of non-survivors $(22 / 25,88.0 \%)$ received NIV, and only 2 of them were performed IMV. Whether to withhold life sustaining therapies in patients with AEIPF is indeed a question. The available data suggested poor outcome for IPF patients who required NIV or IMV, although they temporarily corrected $\mathrm{PaO}_{2} / \mathrm{FiO}_{2}$ and reduced respiratory rate ${ }^{26,27}$. The hospital mortality of AEIPF who required MV was about $90 \%{ }^{28,29}$. A systematic review comprising 9 single-center studies reported that $87.0 \%$ of IPF patients who had received IMV died in hospital ${ }^{30}$. NIV seemed more promising. Rush et al. revealed AEIPF patients who underwent NIV had a lower mortality rate $(30.9 \%)$ compared to those who underwent IMV $(51.6 \%)^{31}$. An early application of NIV when the general conditions of patients were less severe and the prevention of ventilatorassociated pneumonia would benefit the survival ${ }^{4}$. However, ventilator-induced lung injury (VILI), which was an independent predictor for NIV failure ${ }^{32}$, should be acknowledged. In addition, excessive spontaneous effort and deflation injury resulted from NIV also caused lung injury ${ }^{33}$.

Several studies evaluated clinical predictors of mortality in patients with AEIPF, but a universally accepted predicted model did not exist. Akira et al. reported worse survival was associated with diffuse pattern on HRCT $^{34}$. Lower forced vital capacity (FVC) and diffusion capacity for carbon monoxide (DLCO) before AE was observed in non-survivors compared to survivors ${ }^{35}$. Several prognostic biomarkers identified to be related with survival included increased Krebs Von den Lungen-6 (KL-6) $)^{11}$, higher hyaluronan ${ }^{36}$ and anti-heat shock protein 70 autoantibodies ${ }^{37}$. Recently, a staging system with four parameters, serum lactate dehydrogenase (LDH) level, serum KL-6, $\mathrm{PaO}_{2} / \mathrm{FiO}_{2}$, and total extent of abnormal findings on HRCT, was proposed to predict prognosis ${ }^{11}$. Prognostic model which considers pathologic physiology of various organs are acquired to provide guidance on early intervention.

This study had several limitations. First, the data was retrospectively collected from a single-center study. The selection bias was evitable and the small sample size weakened the power of the study. Second, the pulmonary function tests data were incomplete partly because of the critical illness so we could not assess the association between the last pulmonary function data before AE with the mortality.

\section{Conclusion}

The outcome of AEIPF remained poor with a high in-hospital mortality and occurrence of extra-pulmonary organ failure was common. Elevated CRP and extra-pulmonary organ failure were independently associated with hospital mortality. Further investigations were still needed to clarify the association between systemic inflammation and AEIPF. 


\section{Methods}

Patients. Patients diagnosed with AEIPF from July 2014 to September 2018 in Department of Pulmonary and Critical Care Medicine, Nanjing Drum Tower Hospital were enrolled. Electronic medical records were retrospectively reviewed. AEIPF was diagnosed according to the newly updated international guidelines ${ }^{38}$. The criteria were as follows: (1) previous or concurrent diagnosis of IPF with HRCT showing typical usual interstitial pneumonias (UIP) pattern; (2) acute worsening of dyspnea within one month; (3) HRCT showing new bilateral ground-glass opacity and/or consolidation superimposed on a background of UIP pattern and (4) exclusion of cardiac failure or fluid overload. Patients with incomplete data were excluded.

\section{Data collection}

Clinical information was collected, including demographics, smoking history, clinical symptoms, duration from diagnosis of IPF to the occurrence of AE, treatment course and lengths of stay in RICU and hospital. The laboratory data included $\mathrm{PaO}_{2} / \mathrm{FiO}_{2}$, blood routine, CRP, erythrocyte sedimentation rate (ESR), LDH and lymphocyte subset etc. APACHE II score ${ }^{6}$ and SAPS II $^{7}$ in the first $24 \mathrm{~h}$ after admission were calculated. The presence of extra-pulmonary organ failures (coagulation, liver, cardiovascular, central nervous system and renal) were defined according to criteria of SOFA system ${ }^{8}$. Chest HRCTs of all patients in supine position were performed at admission. The newly appeared parenchymal abnormalities were classified into peripheral, multifocal and diffuse patterns based on the distributions ${ }^{34}$. Treatment information included usages and courses of corticosteroids, acetylcysteine, pirfenidone/nintedanib and respiratory support. All patients were administrated with oxygen therapy firstly. NIV was initiated when respiratory rate was more than 30 breaths $/ \mathrm{min}$ or $\mathrm{PaO}_{2} / \mathrm{FiO}_{2}$ was less than $200 \mathrm{mmHg}^{26}$. The primary endpoint was hospital mortality. The length of stay in hospital was calculated from the date of admission to the date of discharge or death.

Statistical analysis. Data was presented as mean with standard deviation for continuous variables or frequencies with percentages for categorical variables. T-test or the Mann-Whitney $U$-test was used for continuous variables. Chi-square test was used for categorical variables. Binary logistic regression analysis was used to evaluate the association between variables and mortality. Survival analysis was estimated using Kaplan-Meier curves and was compared between groups using the log-rank test. For continuous variables, the median was used as cut-off value. $P$ value less than 0.05 was considered statistically significant. All statistical analysis was conducted through IBM SPSS Statistics version 23.0 (SPSS, Inc., Chicago, IL, USA) and Prism 8.0 (GraphPad, Inc., La Jolla, CA, USA).

Ethical considerations. The patient data for this study were recorded by the authors. This study was approved by Ethics Committee of Nanjing Drum Tower Hospital, Clinical College of Nanjing Medical School, Jiangsu, China. Written informed consents were obtained from all subjects and the study was performed in accordance with the relevant guidelines/regulations.

\section{Data availability}

Data can be submitted by the corresponding author in case of a request.

Received: 24 November 2019; Accepted: 8 June 2020

Published online: 01 July 2020

\section{References}

1. Raghu, G. et al. An official ATS/ERS/JRS/ALAT statement: idiopathic pulmonary fibrosis: evidence-based guidelines for diagnosis and management. Am. J. Respir. Crit. Care Med. 183, 788-824. https://doi.org/10.1164/rccm.2009-040GL (2011).

2. Collard, H. R. et al. Acute exacerbations of idiopathic pulmonary fibrosis. Am. J. Respir. Crit. Care Med. 176, 636-643. https://doi. org/10.1164/rccm.200703-463PP (2007).

3. Song, J. W., Hong, S. B., Lim, C. M., Koh, Y. \& Kim, D. S. Acute exacerbation of idiopathic pulmonary fibrosis: incidence, risk factors and outcome. Eur. Respir. J. 37, 356-363. https://doi.org/10.1183/09031936.00159709 (2011).

4. Marchioni, A. et al. Acute exacerbation of idiopathic pulmonary fibrosis: lessons learned from acute respiratory distress syndrome?. Crit Care 22, 80. https://doi.org/10.1186/s13054-018-2002-4 (2018)

5. Papiris, S. A. et al. High levels of IL-6 and IL-8 characterize early-on idiopathic pulmonary fibrosis acute exacerbations. Cytokine 102, 168-172. https://doi.org/10.1016/j.cyto.2017.08.019 (2018).

6. Knaus, W. A., Draper, E. A., Wagner, D. P. \& Zimmerman, J. E. APACHE II: a severity of disease classification system. Crit. Care Med. 13, 818-829 (1985).

7. Le Gall, J. R., Lemeshow, S. \& Saulnier, F. A new Simplified Acute Physiology Score (SAPS II) based on a European/North American multicenter study. JAMA 270, 2957-2963 (1993).

8. Vincent, J. L. et al. The SOFA (Sepsis-related Organ Failure Assessment) score to describe organ dysfunction/failure. On behalf of the Working Group on Sepsis-Related Problems of the European Society of Intensive Care Medicine. Intensive Care Med. 22, 707-710 (1996).

9. Saydain, G. et al. Outcome of patients with idiopathic pulmonary fibrosis admitted to the intensive care unit. Am. J. Respir. Crit. Care Med. 166, 839-842. https://doi.org/10.1164/rccm.2104038 (2002).

10. du Bois, R. M. et al. Ascertainment of individual risk of mortality for patients with idiopathic pulmonary fibrosis. Am. J. Respir. Crit. Care Med. 184, 459-466. https://doi.org/10.1164/rccm.201011-17900C (2011).

11. Kishaba, T., Tamaki, H., Shimaoka, Y., Fukuyama, H. \& Yamashiro, S. Staging of acute exacerbation in patients with idiopathic pulmonary fibrosis. Lung 192, 141-149. https://doi.org/10.1007/s00408-013-9530-0 (2014).

12. Rangappa, P. \& Moran, J. L. Outcomes of patients admitted to the intensive care unit with idiopathic pulmonary fibrosis. Crit. Care Resusc. 11, 102-109 (2009).

13. Kataoka, K. et al. Recombinant human thrombomodulin in acute exacerbation of idiopathic pulmonary fibrosis. Chest 148, 436-443. https://doi.org/10.1378/chest.14-2746 (2015). 
14. Sakamoto, S. et al. Cyclosporin A in the treatment of acute exacerbation of idiopathic pulmonary fibrosis. Intern. Med. 49, 109-115. https://doi.org/10.2169/internalmedicine.49.2359 (2010).

15. Atsumi, K. et al. Prognostic factors in the acute exacerbation of idiopathic pulmonary fibrosis: a retrospective single-center study. Intern. Med. 57, 655-661. https://doi.org/10.2169/internalmedicine.9331-17 (2018).

16. Papiris, S. A. et al. Survival in Idiopathic pulmonary fibrosis acute exacerbations: the non-steroid approach. BMC Pulm. Med. 15, 162. https://doi.org/10.1186/s12890-015-0146-4 (2015).

17. Noble, P. W. et al. Pirfenidone in patients with idiopathic pulmonary fibrosis (CAPACITY): two randomised trials. Lancet 377, 1760-1769. https://doi.org/10.1016/s0140-6736(11)60405-4 (2011).

18. Taniguchi, H. et al. Pirfenidone in idiopathic pulmonary fibrosis. Eur. Respir. J. 35, 821-829. https://doi.org/10.1183/09031 936.00005209 (2010).

19. Azuma, A. et al. Double-blind, placebo-controlled trial of pirfenidone in patients with idiopathic pulmonary fibrosis. Am. J. Respir. Crit. Care Med. 171, 1040-1047. https://doi.org/10.1164/rccm.200404-571OC (2005).

20. McMillan, T. R. et al. Exacerbation of established pulmonary fibrosis in a murine model by gammaherpesvirus. Am. J. Respir. Crit. Care Med. 177, 771-780. https://doi.org/10.1164/rccm.200708-1184OC (2008).

21. Han, M. K. et al. Lung microbiome and disease progression in idiopathic pulmonary fibrosis: an analysis of the COMET study. Lancet Respir. Med. 2, 548-556. https://doi.org/10.1016/s2213-2600(14)70069-4 (2014).

22. Weng, D. et al. The role of infection in acute exacerbation of idiopathic pulmonary fibrosis. Mediators Inflamm 2019, 5160694. https://doi.org/10.1155/2019/5160694 (2019).

23. Luo, L. et al. Clinical predictors of hospital mortality differ between direct and indirect ARDS. Chest 151, 755-763. https://doi. org/10.1016/j.chest.2016.09.004 (2017).

24. Ferring, M. \& Vincent, J. L. Is outcome from ARDS related to the severity of respiratory failure?. Eur. Respir. J. 10, 1297-1300 (1997).

25. Stapleton, R. D. et al. Causes and timing of death in patients with ARDS. Chest 128, 525-532. https://doi.org/10.1378/chest 128.2.525 (2005).

26. Vianello, A. et al. Noninvasive ventilation in the event of acute respiratory failure in patients with idiopathic pulmonary fibrosis. J. Crit. Care 29, 562-567. https://doi.org/10.1016/j.jcrc.2014.03.019 (2014).

27. Mollica, C. et al. Mechanical ventilation in patients with end-stage idiopathic pulmonary fibrosis. Respiration 79, 209-215. https ://doi.org/10.1159/000225932 (2010).

28. Blivet, S. et al. Outcome of patients with idiopathic pulmonary fibrosis admitted to the ICU for respiratory failure. Chest 120, 209-212. https://doi.org/10.1378/chest.120.1.209 (2001).

29. Fumeaux, T., Rothmeier, C. \& Jolliet, P. Outcome of mechanical ventilation for acute respiratory failure in patients with pulmonary fibrosis. Intensive Care Med 27, 1868-1874. https://doi.org/10.1007/s00134-001-1150-0 (2001).

30. S. Mallick, Outcome of patients with idiopathic pulmonary fibrosis (IPF) ventilated in intensive care unit. Respiratory Med. 102, 1355-1359, doi:10.1016/j.rmed.2008.06.003 (2008)

31. Rush, B., Wiskar, K., Berger, L. \& Griesdale, D. The use of mechanical ventilation in patients with idiopathic pulmonary fibrosis in the United States: a nationwide retrospective cohort analysis. Respir Med 111, 72-76. https://doi.org/10.1016/j.rmed.2015.12.005 (2016).

32. Carteaux, G. et al. Failure of noninvasive ventilation for de novo acute hypoxemic respiratory failure: role of tidal volume. Crit Care Med 44, 282-290. https://doi.org/10.1097/ccm.0000000000001379 (2016).

33. Katira, B. H. Ventilator-induced lung injury: classic and novel concepts. Respir. Care 64, 629-637. https://doi.org/10.4187/respc are.07055 (2019)

34. Akira, M., Kozuka, T., Yamamoto, S. \& Sakatani, M. Computed tomography findings in acute exacerbation of idiopathic pulmonary fibrosis. Am. J. Respir. Crit. Care Med. 178, 372-378. https://doi.org/10.1164/rccm.200709-1365OC (2008).

35. Simon-Blancal, V. et al. Acute exacerbation of idiopathic pulmonary fibrosis: outcome and prognostic factors. Respiration 83, 28-35. https://doi.org/10.1159/000329891 (2012).

36. Inokoshi, Y. et al. Clinical significance of serum hyaluronan in chronic fibrotic interstitial pneumonia. Respirology 18, 1236-1243. https://doi.org/10.1111/resp.12144 (2013).

37. Kahloon, R. A. et al. Patients with idiopathic pulmonary fibrosis with antibodies to heat shock protein 70 have poor prognoses. Am. J. Respir. Crit. Care Med.s 187, 768-775. https://doi.org/10.1164/rccm.201203-0506OC (2013).

38. Collard, H. R. et al. Acute exacerbation of idiopathic pulmonary fibrosis. An international working group report. Am J Respir Crit Care Med 194, 265-275, doi:10.1164/rccm.201604-0801CI (2016).

\section{Acknowledgements}

The study was supported by the National Natural Science Foundation of China (81570058), Jiangsu Provincial Medical Talent (ZDRCA2016058), and Jiangsu Social Development Project (BE2017604).

\section{Author contributions}

Y.Z. and Y.Z. collected the data, performed the statistical analysis, searched the literature and prepared the draft of the manuscript. X.Q., Y.X. and H.C. conducted the radiological analysis. J.D. designed the study and revised the manuscript.

\section{Competing interests}

The authors declare no competing interests.

\section{Additional information}

Correspondence and requests for materials should be addressed to J.D.

Reprints and permissions information is available at www.nature.com/reprints.

Publisher's note Springer Nature remains neutral with regard to jurisdictional claims in published maps and institutional affiliations. 
(c) (i) Open Access This article is licensed under a Creative Commons Attribution 4.0 International cc) License, which permits use, sharing, adaptation, distribution and reproduction in any medium or format, as long as you give appropriate credit to the original author(s) and the source, provide a link to the Creative Commons license, and indicate if changes were made. The images or other third party material in this article are included in the article's Creative Commons license, unless indicated otherwise in a credit line to the material. If material is not included in the article's Creative Commons license and your intended use is not permitted by statutory regulation or exceeds the permitted use, you will need to obtain permission directly from the copyright holder. To view a copy of this license, visit http://creativecommons.org/licenses/by/4.0/.

(C) The Author(s) 2020 